\title{
VideoViz - Uma proposta de ferramenta de visualização de logs de interação em players de vídeo
}

\author{
André Luiz de B. Damasceno ${ }^{1}$, Álan L. V. Guedes ${ }^{1}$, Sergio Colcher ${ }^{1}$, Simone D. J. Barbosa ${ }^{1}$ \\ Dilson J. L. Rabêlo ${ }^{2}$, Elza B. Monier ${ }^{2}$ \\ ${ }^{1}$ Pontifícia Universidade Católica do Rio de Janeiro - Rio de Janeiro, RJ - Brasil \\ ${ }^{2}$ UNA-SUS/UFMA - São Luís, MA - Brasil \\ \{andre,alan\}@telemidia.puc-rio.br,\{colcher,simone\}@inf.puc-rio.br,\{dilsonrabelo.unasus,bernardes.elza\}@gmail.com
}

\begin{abstract}
The usage of technologies for content authoring, storage, distribution and presentation supported the arise of Virtual Learning Environments (VLEs) such as Moodle and MOOCs (e.g., Udacity, Coursera, EdX). All students' interaction in these environments can be stored in logs. For instance, video is one of the resources has a greater focus on VLEs. In this paper, we present a state-of-theart overview of interaction analysis on video players. We noticed that teachers would like to know how and when students interact with videos. In order to fill this gap, we propose VideoViz, a tool to analyse logs from interaction on video players.
\end{abstract}

\section{KEYWORDS}

Learning Analytics, Educational Data Mining, MOOC, AVA, e-learning, Video, engagement, behavior, interaction, visualization, tool

\section{INTRODUÇÃO}

Estudantes e professores atualmente utilizam tecnologias que permitem novas maneiras de aprender e ensinar. Apesar da educação a distância $(\mathrm{EaD})$ não ser novidade, havendo registros do século XIX de cursos por correspondência [15], foi a partir da utilização de computadores e da evolução da Internet que permitiu ao aluno participar de forma mais ativa e gerou novos paradigmas nessa modalidade de ensino. Um dos motivos para o crescimento da oferta e demanda de cursos a distância é a conveniência dos alunos determinarem seu ritmo de aprendizagem e a possibilidade deles participarem de cursos sem serem limitados pela distância geográfica. Em particular, o uso de tecnologias de autoria, armazenamento, distribuição e apresentação de conteúdo contribuíram para o surgimento de Ambientes Virtuais de Aprendizagem (AVAs) como MOOCs (Massive Open Online Courses) (e.g., Udacity ${ }^{1}$, Coursera ${ }^{2}, \mathrm{EdX}^{3}$ ).

AVAs como o Moodle ${ }^{4}$ não são restritos aos cursos inteiramente online. Conforme evidenciado por Damasceno et al. [6], há casos em que AVAs são utilizados em cursos presenciais. Nessa modalidade de ensino, também conhecida como blended learning, o professor pode complementar sua aula com os seguintes recursos: postagem de

\footnotetext{
${ }^{1} \mathrm{https}: / /$ udacity.com

${ }^{2}$ https://coursera.org

${ }^{3}$ https://edx.org

${ }^{4}$ https://moodle.org/
}

In: V Workshop "O Futuro da Videocolaboração" (WCT-Video 2018), Salvador, Brasil. Anais do XXIV Simpósio Brasileiro de Sistemas Multimídia e Web: Workshops e Pôsteres. Porto Alegre: Sociedade Brasileira de Computação, 2018.

(c) 2018 SBC - Sociedade Brasileira de Computação.

ISBN 978-85-7669-435-9. notícias, links de materiais da Internet, ferramentas de comunicação (e.g., fórum, chat), quiz e wiki. Outra vantagem dos AVAs é a sua capacidade de coletarem e armazenarem toda interação dos estudantes em formato de logs. Através da análise desses logs é possível obter informações sobre padrões, comportamento, desempenho e características dos estudantes [7, 14].

Educational Data Mining (EDM) e Learning Analytics (LA) são áreas de pesquisa com objetivos e interesses similares na exploração de dados (e.g., logs) em EaD. Ambas utilizam detecção automática de informação e análise humana através de visualizações. Contudo, a EDM utiliza a análise humana como ferramenta para melhorar a detecção automática de informação, enquanto a LA busca potencializar a análise humana através de ferramentas que realizam a detecção automática de informação [17].

Em [6], os autores apresentam um mapeamento sistemático da literatura sobre trabalhos que analisam logs de interação de alunos em AVAs. Além disso, entrevistas conduzidas com professores mostram quais informações dos alunos eles consideram relevantes e quais são os requisitos necessários para ajudá-los na análise dessas informações. Os autores também observaram que a maioria dos artigos encontrados visam identificar padrões de comportamento e performance dos estudantes. Entre esses trabalhos, foram encontrados 20 ferramentas para auxiliar o professor na análise de logs. Porém, nenhuma delas preenche todos os requisitos mencionados pelos professores.

Os professores consideram importante extrair informação a partir da interação e feedbacks dos alunos nos sistemas de EaD [6]. Contudo, a maioria dos professores obtêm informações limitadas, como dados de último acesso dos estudantes e postagens dos alunos nos fóruns dos AVAs. Em geral, os professores não recebem treinamento para extrair informações dos logs. Por isso, eles sentem necessidade de ferramentas que analisam logs de acesso e interação dos estudantes, tanto com os materiais, quanto com os recursos dos AVAs como fórum, player de vídeo, quiz e ebooks.

Nesse contexto, este artigo apresenta nossa pesquisa em andamento sobre análise de logs de vídeos. Mais precisamente, apresentamos uma visão geral do estado da arte de análise dos logs de interação em players de vídeos. Em particular, essa visão geral também apresenta o que professores gostariam de saber, como quando e de que forma os alunos interagem com os vídeos. Com o objetivo de preencher a lacuna de ferramentas para auxílio na análise desses logs, apresentamos uma proposta de ferramenta chamada VideoViz.

O restante deste artigo está organizado como a seguir. A Seção 2 discute trabalhos que compartilham nossa motivação de análise de vídeos. A Seção 3 apresenta nossa proposta de ferramenta de 
análise de vídeos chamada VideoViz. Por fim, a Seção 4 discute considerações finais e trabalhos futuros.

\section{ESTADO DA ARTE}

Vídeo é um dos recursos que tem ganhado destaque nos cursos de $\mathrm{EaD}$ [16] e o streaming é a forma dominante de acesso a eles [10]. Segundo alguns professores, a videoaula é o formato de conteúdo que os alunos mais gostam de acessar, porque além de permitir que a explicação de um assunto possa ser vista mais de uma vez, em muitas vezes é o primeiro recurso utilizado para entender um determinado assunto [6]. Portanto, pesquisas como autoria de videoaula e interações com players de vídeo têm tido relevância na área de Informática na Educação (IE). Nesta seção apresentamos o estado da arte das pesquisas em IE envolvendo vídeo, organizadas nas seguintes perspectivas: 2.1 análise de engajamento em vídeos; e 2.2 visualização de informação.

\subsection{Análise de engajamento em vídeos}

Em IE, engajamento é um termo associado a mais de um significado (e.g., número de materiais acessados, acessos aos AVAs, atividades realizadas, interações e postagens nos fóruns). Contudo, em pesquisas com vídeos engajamento é associado a retenção ou tempo de visualização do vídeo [1, 8]. Um dos principais problemas identificados pelos professores é o engajamento dos alunos nos vídeos, pois geralmente eles não assistem uma videoaula inteira $[8,9,12]$. A retenção dos alunos nos vídeos é um dado importante a ser levado em consideração, pois o tempo que os alunos passam assistindo uma videoaula está correlacionado com o desempenho obtido por eles nos cursos [13,21].

Muitos professores possuem experiência no desenvolvimento de material educacional como vídeos, slides e áudios. Por isso, em muitos cursos eles acabam desempenhando também o papel de autores de conteúdo [6]. Segundo eles, há indícios de que a retenção dos alunos nos vídeos está relacionada com a autoria da videoaula. Existem pelo menos 13 estilos diferentes de apresentação dessas videoaulas [4], e algumas pesquisas relatam que o formato de slides não obtêm um bom resultado [6,12]. Corroborando com esses indícios, Guo et al. [8] verificaram que videoaulas no formato Khan academy e Talking-head são os que mais favorecem o engajamento dos alunos, filmagens de aulas presenciais têm um baixo índice de retenção dos alunos e as videoaulas que passam por um processo de pré-produção apresentam resultados melhores na permanência dos alunos. Além disso, os alunos se engajam mais com vídeos filmados informalmente com o professor sentado em sua mesa, quando comparados com vídeos produzidos no formato de produção de alto custo de TV [8].

Em entrevistas [6], alguns professores citaram como fatores que influenciam o aluno a assistir uma videoaula: (i) a proximidade da prova, (ii) a produção e a edição do vídeo, (iii) o formato de exibição do conteúdo e (iv) a maneira como o professor se apresenta e se expressa na videoaula, pois professores que falam devagar ocasionam um baixo engajamento nas videoaulas [8]. A duração do vídeo foi mais um fator mencionado por esses professores. Segundo eles, o aluno não costuma assistir vídeos longos e, quando acessa, não assiste o vídeo todo. Contudo, não houve consenso sobre a duração ideal de uma videoaula (variando de 5 a 30 minutos de duração máxima). A variação do engajamento conforme a duração do vídeo também foi discutida na literatura. McGowan et al. [12] observaram uma correlação negativa entre a retenção de audiência e a duração do vídeo. E Guo et al. [8] observaram que os alunos se engajam menos em exercícios que sucedem vídeos longos.

O conteúdo apresentado também tem relação direta com o engajamento do aluno. Der Sluis et al. [20] observaram que a permanência dos estudantes aumenta em trechos com muitas informações ou informações complexas. Alguns professores notaram que há pouco engajamento dos alunos em vídeos mais teóricos, sem demonstração ou resolução de exercícios [6]. Em relação a isso, Guo et al. [8] verificaram que os alunos assistem mais tutoriais múltiplas vezes, e McGowan et al. [12] notaram que os vídeos de teoria retêm menos alunos do que os vídeos de demonstração de código. $\mathrm{Na}$ área de informática, McGowan et al. [12] observaram ainda que videoaulas que apresentam o código pronto, com explicação de cada trecho de código pelo professor, possuem mais engajamento dos alunos quando comparados com videoaulas que exibem o professor digitando e explicando o código.

\subsection{Visualização de informação}

Em MOOCs, os alunos passam mais tempo assistindo vídeos $[11,16]$. Isso é um dos fatores que fazem com que a interação dos estudantes com os players de vídeo seja uma das fontes de dados utilizadas nas pesquisas de clusterização e predição de performance dos estudantes $[2,5]$. Além disso, quando os professores foram perguntados sobre o que eles gostariam de saber com base na interação dos alunos com as videoaulas [6], a maioria respondeu querer visualizar informações de acesso aos vídeos, como estatísticas de visualização de certos trechos dos vídeos, quais trechos foram pulados e quais trechos os alunos assistiram mais de uma vez. Segundo eles, essas informações podem sinalizar ao professor o que os alunos acharam relevante e sobre quais trechos os alunos tiveram mais dúvidas.

Guo e Reinecke [9] trouxeram evidências de que a interação na timeline dos players de vídeos pode ser utilizada como um indicador de performance dos estudantes. Em outras palavras, quanto maior o número de interações na timeline, maior a probabilidade do estudante ter uma boa performance no curso. No contexto de cursos de inglês, um padrão de comportamento observado com relação à interação com a timeline foi que os estudantes que não possuem fluência no idioma realizam mais ações de play, pause e seek do que os estudantes fluentes $[18,19]$.

Um resultado empírico observado por professores é que pessoas mais velhas interagem nos AVAs de forma diferente das pessoas mais novas [6]. Isso é corroborado com os resultados obtidos por Guo e Reinecke [9], que relatam que os estudantes mais velhos tendem a obter melhor performance e a realizar mais backjumps ${ }^{5}$ na timeline dos players de vídeos do que os estudantes mais novos. Além disso, eles também observaram que os países que possuem maior razão entre o número de estudantes por professor, em salas de aula presenciais, são os que correspondem aos menores índices de backjumps.

Podemos encontrar na literatura ferramentas de visualização de logs de interação (e.g., de eventos do tipo play, pause e seek) por trechos de uma videoaula [5]. Como evolução dessa ferramenta,

\footnotetext{
${ }^{5}$ Mover o cursor da timeline para um instante de tempo anterior ao atual
} 
Chen et al. [3] apresentam uma visualização para ajudar os professores a identificarem os alunos que desistiram do curso, com base em dados de acesso aos vídeos. Contudo, os professores reportaram problemas de interpretação da visualização e de usabilidade da ferramenta.

Encontramos um único trabalho para identificar padrões de interação no player de vídeo que resultam em uma boa performance no curso [2]. O trabalho apresenta uma ferramenta que agrupa (em clusters) os alunos pelas suas interações e gera uma visualização que relaciona os clusters com as notas obtidas pelos alunos. Entretanto, foi observado que clusters que apresentam o mesmo padrão de interação possuem alunos com performances satisfatória e insatisfatória no curso. Além disso, quando a ferramenta foi testada pelos professores, eles reportaram problemas no entendimento da visualização, mesmo depois de terem passado por um tutorial.

\section{PROPOSTA DE TRABALHO}

Como ressaltado na seção anterior, há uma lacuna de ferramentas para auxiliar os professores na análise de logs de interação dos alunos. Portanto, este trabalho apresenta uma proposta de ferramenta para análise de interações com os players de vídeo, chamada VideoViz. A utilização de técnicas de agrupamento (clustering) de dados na VideoViz pode fornecer feedbacks ao autor do conteúdo sobre o comportamento dos usuários, assim como gerar insights para o autor sobre quais os trechos do vídeo são relevantes e qual o melhor dia da semana para postar conteúdo, levando em consideração critérios como: quantidade de acessos e perfil de usuários. No caso de autores, que geralmente são professores, a ferramenta pode apresentar insights sobre trechos do video em que ocorrem dúvidas e comparar a interação nos players de vídeo entre os alunos com performance satisfatória e os com baixo rendimento no curso.

A VideoViz pode ser utilizada tanto em AVAs como em repositório de vídeos (RVs). RVs como os fornecidos pela RNP (e.g., Video@RNP6 e Videoaula@RNP ${ }^{7}$ ), e AVAs como os mantidos pela UNA-SUS/UFMA ${ }^{8}$, permitem que usuários acessem conteúdos de diversas áreas (e.g., humanas, exatas, saúde). Ao todo, ambos os RVs da RNP possuem cerca de 27 mil vídeos, com mais de $20 \mathrm{mil}$ acessos por mês. Já o AVA da UNA-SUS/UFMA registra mais de 66 mil acessos por mês nos seus mais de 800 recursos utilizados nos cursos (e.g., vídeos, ebooks, slides).

Entretanto, RVs como VideoRNP e Videoaula@RNP têm uma limitação para visualização de informação com base nos logs de interação, pois os usuários podem acessar os vídeos sem terem feito login. Logo, as visualizações para esses RVs não podem correlacionar interações com o perfil dos usuários (e.g., idade, escolaridade) ou performance (i.e., notas) deles nos cursos que utilizam seus vídeos. $\mathrm{O}$ que é possível nos RVs é associar as interações de cada usuário com o identificador de sessão do browser e apresentar visualizações sobre o acesso ao vídeo (e.g., ações, data e região). Já em AVAs como o da UNA-SUS/UFMA, além de visualizar as mesmas informações de RVs, é possível gerar clusters de perfil, escolaridade e performance do aluno. Podemos observar essas informações resumidas na Tabela 1.

\footnotetext{
${ }^{6}$ http://video.rnp.br

${ }^{7}$ http://videoaula.rnp.br

${ }^{8}$ http://avaunasus.ufma.br
}

\begin{tabular}{|l|c|c|}
\hline Visualização de clusters de interações por: & RVs & AVAs \\
\hline sessão do browser & $\mathrm{x}$ & $\mathrm{x}$ \\
\hline data (e.g., dias da semana, mês, semestre) & $\mathrm{x}$ & $\mathrm{x}$ \\
\hline região de origem (e.g., cidades, estado, país) & $\mathrm{x}$ & $\mathrm{x}$ \\
\hline perfil (e.g., idade, escolaridade) & & $\mathrm{x}$ \\
\hline performance em curso (e.g., notas) & & $\mathrm{x}$ \\
\hline
\end{tabular}

Tabela 1: Possibilidades de análise de logs em RVs e AVAs

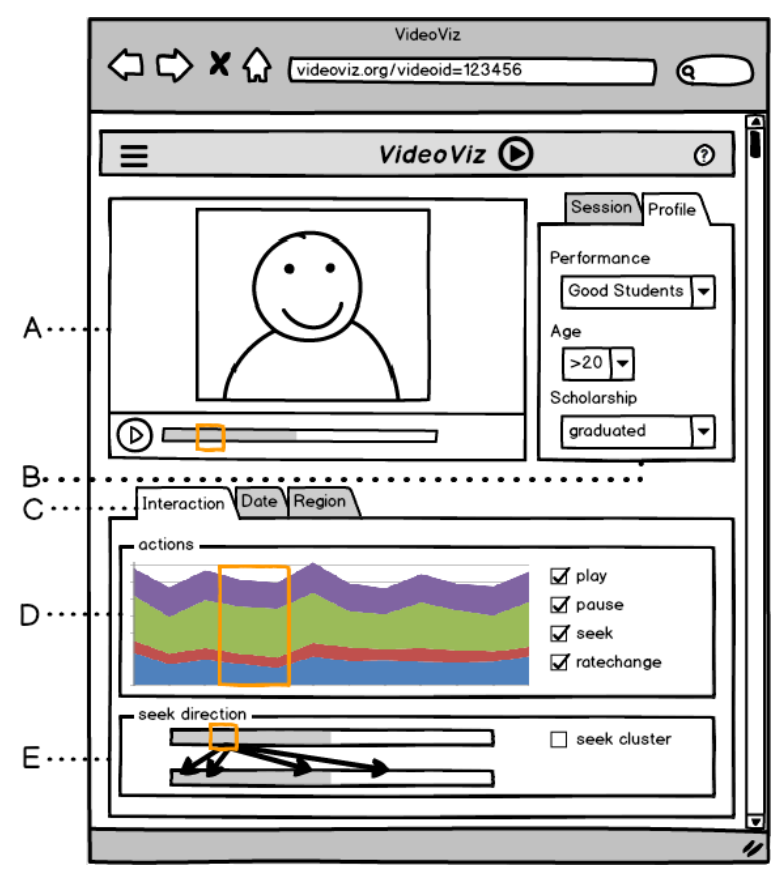

Figura 1: Esboço da ferramenta VideoViz

A VideoViz visa a apresentar para RVs e AVAs informações de padrões de acesso e de interação nos vídeos, por meio de técnicas visualização de dados. Entretanto, as visualizações são diferenciadas dada a origem do vídeo (RVs ou AVAs). A Figura 1 ilustra um mockup da ferramenta, o qual detalhamos a seguir: (A) área do player que reproduz o vídeo, cuja navegação é utilizada como filtro de logs a serem analisados, ou seja, o instante de tempo do vídeo é utilizado como referência nas demais visualizações; (B) painel de indicação de como o usuário da ferramenta deseja que visualização seja correlacionada com dados de perfil como performance, idade e escolaridade. Caso seja uma RV, essas correlações são limitadas à sessão do browser; (C) seletor de diferentes visualizações, presentes tanto em RVs quanto em AVAs. Nesse caso, a ferramenta apresenta opções de visualização sobre as interações no player. Outros tipos de visualização são por data e região de onde o usuário está acessando: (D) mostra um gráfico de área da distribuição das ações (e.g., play, pause, seek, ratechange) executadas pelo usuário no player durante a execução do vídeo; (E) exibe a visualização dos instantes de tempo de destino da maioria das ações de seek realizadas pelos usuários. Em particular, nessa visualização, a ferramenta também apresenta clusters de movimentação do marcador de tempo do vídeo. Isso é útil para indicar trechos repetidamente assistidos, que podem indicar 
trechos com mais relevância ou de dúvidas. Além disso, o cluster também pode indicar trechos que são frequentemente pulados, que podem indicar trechos do vídeo a serem resumidos ou removidos.

\section{CONCLUSÃO}

Este trabalho apresenta uma visão geral do estado da arte de análise dos logs de interação em players de vídeos. Com o objetivo de preencher a lacuna de ferramentas para auxílio na análise desses logs, apresentamos o VideoViz como sendo uma proposto de ferramenta para ser utilizada por AVAs e RVs. Vale ressaltar que, apesar da ferramenta considerar apenas logs de interação em vídeos, é possível estendê-la para analisar logs de outros recursos (e.g., fórum, quiz, ebooks) e criar modelos de predição de abandono e performance do aluno.

A proposta faz parte de uma pesquisa em andamento. Por isso, existe a necessidade de refinamentos. Como trabalho futuro propomos a utilização de técnicas de design participativo para inclusão de autores de conteúdo (e.g., professor) no centro do processo de desenvolvimento da ferramenta. Além disso, buscamos investigar se há melhora no desempenho da turma quando os professores utilizam ferramentas de clustering e predição de desempenho dos alunos.

\section{AGRADECIMENTOS}

Nós agradecemos a instituição UNA-SUS/UFMA pela colaboração e parceira, e a Dalai Ribero pelas discussões sobre esse trabalho. Além disso, o presente trabalho foi realizado com apoio da Coordenação de Aperfeiçoamento de Pessoal de Nível Superior - Brasil (CAPES) - Código de Financiamento 001. Agradecemos também a CNPq pelo financiamento (projeto \#309828/2015-5).

\section{REFERÊNCIAS}

[1] Miguel L. Bote-Lorenzo and Eduardo Gómez-Sánchez. 2017. Predicting the decrease of engagement indicators in a MOOC. In Proceedings of the Seventh International Conference on Learning Analytics \& Knowledge (LAK '17). ACM, New York, NY, USA, 143-147. https://doi.org/10.1145/3027385.3027387

[2] Qing Chen, Yuanzhe Chen, Dongyu Liu, Conglei Shi, Yingcai Wu, and Huamin Qu. 2016. PeakVizor: Visual Analytics of Peaks in Video Clickstreams from Massive Open Online Courses. IEEE Transactions on Visualization and Computer Graphics 22, 10 (oct 2016), 2315-2330. https://doi.org/10.1109/TVCG.2015.2505305

[3] Yuanzhe Chen, Qing Chen, Mingqian Zhao, Sebastien Boyer, Kalyan Veeramachaneni, and Huamin Ou. 2017. DropoutSeer: Visualizing learning patterns in Massive Open Online Courses for dropout reasoning and prediction. 2016 IEEE Conference on Visual Analytics Science and Technology, VAST 2016 - Proceedings (2017), 111-120. https://doi.org/10.1109/VAST.2016.7883517

[4] Konstantinos Chorianopoulos. 2018. A Taxonomy of Asynchronous Instructional Video Styles. The International Review of Research in Open and Distributed Learning 19, 1 (feb 2018). https://doi.org/10.19173/irrodl.v19i1.2920

[5] Conglei Shi, Siwei Fu, Oing Chen, and Huamin Ou. 2015. VisMOOC: Visualizing video clickstream data from Massive Open Online Courses. In 2015 IEEE Pacific Visualization Symposium (PacificVis), Vol. 2015-July. IEEE, 159-166. https://doi. org/10.1109/PACIFICVIS.2015.7156373

[6] André Luiz de Brandão Damasceno, Dalai dos Santos Ribeiro, Sérgio Colcher, and Simone D. J. Barbosa. 2018. Entrevistas e mapeamento sistemático sobre análise de $\log s$ de interação de alunos em ambientes virtuais de aprendizagem. (2018) 11 pages. ftp://ftp.inf.puc-rio.br/pub/docs/techreports/18 08 damasceno.pdf

[7] Ashish Dutt, Maizatul Akmar Ismail, and Tutut Herawan. 2017. A Systematic Review on Educational Data Mining. IEEE Access 5 (2017), 15991-16005. https: //doi.org/10.1109/ACCESS.2017.2654247

[8] Philip J. Guo, Juho Kim, and Rob Rubin. 2014. How video production affects student engagement: an empirical study of MOOC videos. In Proceedings of the first ACM conference on Learning @ scale conference (L@S '14). ACM Press, 41-50. https://doi.org/10.1145/2556325.2566239 arXiv:arXiv:1011.1669v3

[9] Philip J. Guo and Katharina Reinecke. 2014. Demographic differences in how students navigate through MOOCs. In Proceedings of the first ACM conference on
Learning @ scale conference (L@S '14). ACM Press, New York, New York, USA, 21-30. https://doi.org/10.1145/2556325.2566247 arXiv:arXiv:1011.1669v3

[10] René F Kizilcec, Chris Piech, and Emily Schneider. 2013. Deconstructing disengagement: analyzing learner subpopulations in massive open online courses. In Proceedings of the Third International Conference on Learning Analytics and Knowledge (LAK '13). ACM Press, New York, New York, USA, 170. https://doi.org/10.1145/2460296.2460330

[11] Vitomir Kovanović, Srećko Joksimović, Dragan Gašević, James Owers, AnneMarie Scott, and Amy Woodgate. 2016. Profiling MOOC Course Returners: How Does Student Behavior Change Between Two Course Enrollments?. In Proceedings of the Third (2016) ACM Conference on Learning @ Scale (L@S '16). ACM Press, New York, New York, USA, 269-272. https://doi.org/10.1145/2876034. 2893431

[12] Aidan McGowan, Philip Hanna, and Neil Anderson. 2016. Teaching Programming: Understanding Lecture Capture YouTube Analytics. In Proceedings of the 2016 ACM Conference on Innovation and Technology in Computer Science Education (ITiCSE '16). ACM Press, New York, New York, USA, 35-40. https://doi.org/10.1145/2899415.2899421

[13] Jiezhong Qiu, Jie Tang, Tracy Xiao Liu, Jie Gong, Chenhui Zhang, Qian Zhang, and Yufei Xue. 2016. Modeling and Predicting Learning Behavior in MOOCs. In Proceedings of the Ninth ACM International Conference on Web Search and Data Mining (WSDM '16). ACM, New York, NY, USA, 93-102. https://doi.org/10.1145/ 2835776.2835842

[14] Cristóbal Romero and Sebastián Ventura. 2010. Educational Data Mining: A Review of the State of the Art. IEEE Transactions on Systems, Man, and Cybernetics, Part C (Applications and Reviews) 40, 6 (Nov 2010), 601-618. https://doi.org/10. 1109/TSMCC.2010.2053532

[15] Terezinha Saraiva. 1996. Educação a distância no Brasil: lições da história. Em Aberto 16, 70 (abr 1996), 17-27. https://doi.org/10.24109/2176-6673.emaberto. $16 \mathrm{i} 70.2076$

[16] Daniel T. Seaton, Yoav Bergner, Isaac Chuang, Piotr Mitros, and David E Pritchard. 2014. Who does what in a massive open online course? Commun. ACM 57, 4 (apr 2014), 58-65. https://doi.org/10.1145/2500876

[17] George Siemens and Ryan S. J. d. Baker. 2012. Learning Analytics and Educational Data Mining: Towards Communication and Collaboration. In Proceedings of the 2Nd International Conference on Learning Analytics and Knowledge (LAK '12). ACM, New York, NY, USA, 252-254. https://doi.org/10.1145/2330601.2330661

[18] Selen Türkay, Hadas Eidelman, Yigal Rosen, Daniel Seaton, Glenn Lopez, and Jacob Whitehill. 2017. Getting to Know English Language Learners in MOOCs: Their Motivations, Behaviors, and Outcomes. In Proceedings of the Fourth (2017) ACM Conference on Learning @ Scale (L@S '17). ACM, New York, NY, USA, 209-212. https://doi.org/10.1145/3051457.3053987

[19] Judith Uchidiuno, Jessica Hammer, Evelyn Yarzebinski, Kenneth R. Koedinger, and Amy Ogan. 2017. Characterizing ELL Students' Behavior During MOOC Videos Using Content Type. In Proceedings of the Fourth (2017) ACM Conference on Learning@ @cale (L@S '17). ACM, New York, NY, USA, 185-188. https: //doi.org/10.1145/3051457.3053981

[20] Frans Van der Sluis, Jasper Ginn, and Tim Van der Zee. 2016. Explaining Student Behavior at Scale: The Influence of Video Complexity on Student Dwelling Time. In Proceedings of the Third (2016) ACM Conference on Learning @ Scale (L@S '16). ACM Press, New York, New York, USA, 51-60. https://doi.org/10.1145/2876034. 2876051

[21] Hongyan Wang, Xingwei Hao, Wenjiang Jiao, and Xingwu Jia. 2016. Causal Association Analysis Algorithm for MOOC Learning Behavior and Learning Effect. In 2016 IEEE 14th Intl Conf on Dependable, Autonomic and Secure Computing, 14th Intl Conf on Pervasive Intelligence and Computing, 2nd Intl Conf on Big Data Intelligence and Computing and Cyber Science and Technology Congress(DASC/PiCom/DataCom/CyberSciTech). IEEE, 202-206. https: //doi.org/10.1109/DASC-PICom-DataCom-CyberSciTec.2016.53 